\title{
Modelling of Shaft Orbiting with 3-D Solid Finite Elements
}

\author{
J. YU ${ }^{\mathrm{a}, *}$ A. CRAGGS ${ }^{\mathrm{b}}$ and A. MIODUCHOWSKI ${ }^{\mathrm{b}}$ \\ ${ }^{a}$ Bently Rotor Dynamics Research Corporation, P.O. Box 2529, Minden, NV 89423, USA; \\ ${ }^{b}$ Department of Mechanical Engineering, University of Alberta, Edmonton, AB T6G 2G8, Canada
}

(Received in final form 29 May 1998)

\begin{abstract}
A 3-D solid finite element model which can include bending, torsional, axial and other motions is proposed to analyse dynamic responses of shafts. For uniform shafts, this model shows consistency with beam theories when bending vibration is examined. For non-uniform shafts such as tapered ones, however, this model gives much more reliable and accurate results than beam theories which use an assumption that plane sections remain plane. Reduction procedures can be applied which involve only small matrix operations for such a system with a large number of degrees of freedom. The equations of motion have been consistently derived in a rotating frame. Shaft orbiting motion is then defined in this frame, giving a clear view of its trajectories. Forced responses due to excitation in the rotating frame have been examined to find some characteristics of the orbiting shaft. Resonant orbiting frequencies, i.e., natural frequencies of rotating shafts, can be determined in terms of the rotating or fixed frame. Trajectories of transverse displacements have been found to be varying with the forcing frequencies. At resonance, a uniform shaft will only have forward or backward orbiting motion with circular orbits. For other forcing frequencies, however, even a uniform shaft could present both forward and backward orbiting motions with non-circular orbits at different locations along its length. It is anticipated that modelling of shaft orbiting in the rotating frame with the proposed 3-D solid finite elements will lead to accurate dynamic stress evaluation.
\end{abstract}

Keywords: Rotordynamics, 3-D solid finite elements, Forward and backward orbiting, Forced response, Reduction procedure

\section{INTRODUCTION}

Shafts are analysed more accurately by a distributed parameter model than by a lumped parameter model, though the former may involve a simpler solving procedure. Due to shaft configurations, the continuous bar theory has often been used to formulate the equations of motion in terms of one spatial variable along its neutral axis and time. When bending vibration is analysed, the bar is usually called the beam in which transverse displacements and their slopes are of interest. For

\footnotetext{
* Corresponding author. Tel.: (702)782-3611. Fax: (702)782-9236. E-mail: john.yu( $\propto$ ) bently.com.
} 
torsional and axial vibrations, twist angles of cross sections and axial displacements are examined, respectively. The Timoshenko beam theory (Timoshenko, 1922) has been used extensively to model the shaft bending behaviour with a finite element (FE) technique (Nelson, 1980; Rouch and Kao, 1979; Akella and Craggs, 1986). However, as indicated by Senthilnathan and Lee (1992), this beam theory may only be adequate to calculate the first few natural frequencies of thick beams with a shear factor of around 5/6, not adequate to evaluate stresses of a vibrating beam. It is also known that this beam theory is based on an assumption that plane sections remain plane during the deformation. This assumption is violated for tapered beams (Vest and Darlow, 1990).

A few researchers have tried solid FE approaches instead of beam elements to model rotors. Geradin and Kill (1984) first derived FE equations for both beam-like and axisymmetric structures. In beam-like structures an assumption that cross-sections are conserved and remain orthogonal to the neutral axis after deformation was made. Stephenson et al. (1989) and Stephenson and Rouch (1993) utilized axisymmetric elements to analyse bending vibrations of rotors. Genta and Tonoli (1996) have also used axisymmetric elements to model discs in the rotordynamic analysis. In order to carry out easily dynamic analysis involving bending, torsional, axial and other possible modes, and coupled modes among them, and to model non-circular cross sections, distributed density and distributed hydrodynamic pressure, the authors have employed an elastic shaft model with 3-D solid finite elements. Earlier work (Yu and Craggs, 1995a; 1997) has shown that it is a successful and effective approach. Since the 3-D solid FE approach is used instead of conventional beam element theories to model the dynamic response of a rotating shaft, much more degrees of freedom may have to be adopted. Fortunately, for this chain-like structure, reduction procedures such as a continuous coordinate condensation (Craggs and Eckert, 1992) or a transfer matrix method (Yu and Craggs, 1995b) can be imple- mented to obtain the modes of interest or forced responses without loss of accuracy.

In this paper, rotating hollow shafts are modelled using 3-D solid finite elements in the rotating frame. It should be noted that if the fixed frame were to be used, a displacement function would involve both rigid and elastic components. Thus unknown displacements would no longer be small (Choi et al., 1992) and the corresponding FE formulation would be more complicated. The potential energy would involve rigid body motion, thus yielding time-dependent stiffness. However, when the rotating reference frame is introduced, displacement functions involve only an elastic motion. This allows one to view the complicated dynamic behaviour of the rotating shaft as though it were simply oscillating in the rotating frame like a nonrotating shaft.

Recently, Vance (1988), Muszynska (1996) and Muszynska et al. (1996) found the phenomenon of forward and backward orbiting coexistence along a rotor in the fixed reference frame. This paper will discuss the shaft orbiting in the rotating frame. It was found that if harmonic bending excitations are applied in the rotating frame, a shaft would generally move counterclockwise at one section, and clockwise at another section. However, if the forcing frequency is equal to the natural frequency of the rotating shaft, i.e., the resonant forward or backward orbiting frequency, the shaft will only present either pure forward or pure backward orbiting. Until now this phenomenon, which could exist in the rotating frame at certain excitations, has never been indicated. An explanation will be given in addition to numerical examples.

It should be noted that simple beam theories may also give an insight into the nature of rotating shafts including the above-mentioned unusual coexistence of forward and backward orbiting. However, 3-D solid FE modelling can give more accurate and reliable results in addition to revealing some phenomena. It is expected that dynamic stresses could be evaluated accurately in terms of relative elastic displacements in the rotating frame. 


\section{FINITE ELEMENT MODELLING}

Instead of beam theories, a 3-D continuum model is employed to simulate the dynamic response of a rotating shaft, which is assumed to be rotating about its undeformed centroidal line with rotational speed $\Omega$. The motion of the body is divided into the rigid body motion, which defines the rotating frame, and the small elastic motion. Gyroscopic effects are automatically included with this consistent modelling.

Since displacements are measured relatively to the rotating frame, an assumption of small strains and displacements is valid, which facilitates an elastic dynamic analysis using 3-D solid FE techniques. Shaft elements or superelements are composed of several basic continuum finite elements. Huge eigenvalue problems can be avoided when the abovementioned procedures are implemented to obtain the responses in the frequency ranges of interest.

\subsection{Dynamic Model}

An arbitrary particle in the shaft, depicted in Fig. 1, will have both rigid body and elastic displacements. The frame $(O X Y Z)$ is fixed. The $Z$-axis coincides with the undeformed centroidal line of the shaft. The rotating frame $(O x y z)$ with unit basis vectors $i$, $\boldsymbol{j}$, and $\boldsymbol{k}$, has the rigid body motion of the shaft. It is obtained from the fixed frame by a rotation of angle $\Omega t$ about the $Z$-axis. Only an elastic motion

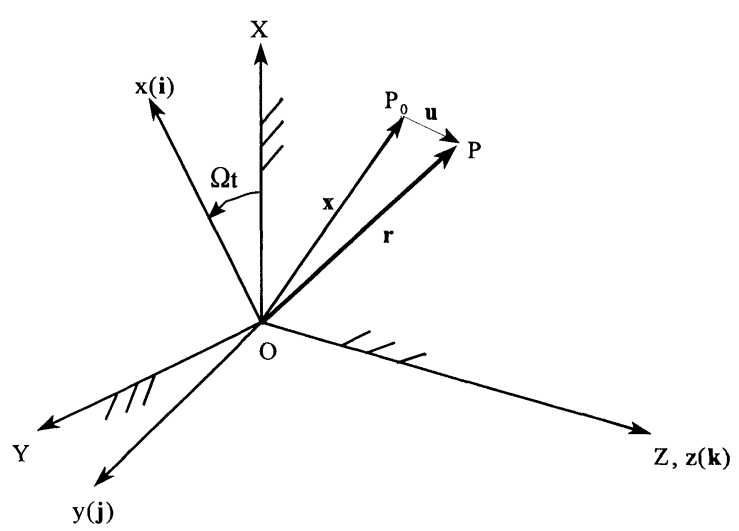

FIGURE 1 Reference frames and particle positions. needs to be examined for all particles in the shaft. The material particle position $P_{0}$ in the undeformed configuration will move to the position $P$, all in the rotating frame.

For the particle with a position vector $\boldsymbol{x}$, its displaced position vector $r$ involving a displacement $\boldsymbol{u}$ can be given by

$$
\boldsymbol{r}=\boldsymbol{x}+\boldsymbol{u},
$$

where

$$
\boldsymbol{x}=x \boldsymbol{i}+y \boldsymbol{j}+z \boldsymbol{k}, \quad \boldsymbol{u}=u \boldsymbol{i}+v \boldsymbol{j}+w \boldsymbol{k} .
$$

The particle velocity can be expressed as

$$
\dot{\boldsymbol{r}}=\left.\frac{\partial}{\partial t} \boldsymbol{r}(\boldsymbol{x}, t)\right|_{\boldsymbol{x}} .
$$

Thus the kinetic energy for any part of the rotating shaft body is given by

$$
\begin{aligned}
T=\frac{1}{2} \iiint & \rho \dot{\boldsymbol{r}} \cdot \dot{\boldsymbol{r}} \mathrm{d} x \mathrm{~d} y \mathrm{~d} z \\
=\frac{1}{2} \iiint & \rho\left[\dot{u}^{2}+\dot{v}^{2}+\dot{w}^{2}+\Omega^{2}\left(u^{2}+v^{2}\right)\right. \\
& +2 \Omega^{2}(y v+x u)+2 \Omega(u \dot{v}-v \dot{u}) \\
& \left.+\Omega^{2}\left(x^{2}+y^{2}\right)+2 \Omega(x \dot{v}-y \dot{u})\right] \mathrm{d} x \mathrm{~d} y \mathrm{~d} z,
\end{aligned}
$$

where $\rho$ is the density of the concerned part. The potential or strain energy for this part can be written as

$$
\begin{aligned}
V= & \frac{E}{2(1+\nu)} \iiint\left[\frac{\nu}{1-2 \nu}\left(\frac{\partial u}{\partial x}+\frac{\partial v}{\partial y}+\frac{\partial w}{\partial z}\right)^{2}\right. \\
& +\left(\frac{\partial u}{\partial x}\right)^{2}+\left(\frac{\partial v}{\partial y}\right)^{2}+\left(\frac{\partial w}{\partial z}\right)^{2} \\
& +\frac{1}{2}\left(\frac{\partial w}{\partial y}+\frac{\partial v}{\partial z}\right)^{2}+\frac{1}{2}\left(\frac{\partial u}{\partial z}+\frac{\partial w}{\partial x}\right)^{2} \\
& \left.+\frac{1}{2}\left(\frac{\partial v}{\partial x}+\frac{\partial u}{\partial y}\right)^{2}\right] \mathrm{d} x \mathrm{~d} y \mathrm{~d} z
\end{aligned}
$$

where $E$ is Young's modulus and $\nu$ is Poisson's ratio. Note that if expressed in the fixed frame, the potential energy, which involves rigid body motion of shaft spinning, will be time-dependent. 


\subsection{3-D Solid Shaft Element Formulations}

A shaft is divided along its axial line into a series of short shaft elements which are defined as superelements. Each superelement consists of four basic continuum elements whose geometry is a quarterannulus, as shown in Fig. 2(a). One or more superelements replace a conventional beam or bar element for the shaft modelling. Use of the superelement allows one to treat it as a shaft element, so that some condensation procedures (Craggs and Eckert, 1992; Yu and Craggs, 1995b) can be employed to facilitate computational operations without any loss of accuracy.

For a basic continuum element as shown in Fig. 2(b), the real coordinates of its geometry can be defined in terms of local coordinates $(\xi, \eta, \zeta)$ in the following form:

$$
\begin{aligned}
& x=\{g\}^{\mathrm{T}}[T]^{-1}\left\{x_{i}\right\}, \\
& y=\{g\}^{\mathrm{T}}[T]^{-1}\left\{y_{i}\right\}, \\
& z=\{g\}^{\mathrm{T}}[T]^{-1}\left\{z_{i}\right\},
\end{aligned}
$$

where

$$
\begin{aligned}
\{g\}^{\mathrm{T}} & =\left[\{h\}^{\mathrm{T}}, \zeta\{h\}^{\mathrm{T}}\right], \\
\{h\}^{\mathrm{T}} & =\left[1, \xi, \eta, \xi^{2}, \xi \eta, \eta^{2}, \xi^{2} \eta, \xi \eta^{2}\right], \\
{[T] } & =\text { coefficient matrix }, \\
\left\{x_{i}\right\},\left\{y_{i}\right\},\left\{z_{i}\right\} & =\text { coordinates at nodes. }
\end{aligned}
$$
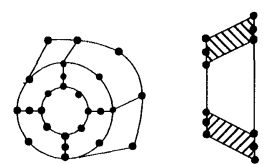

( a )
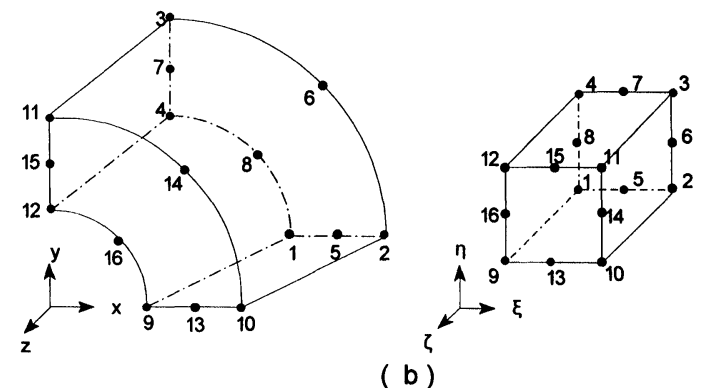

FIGURE 2 Shaft modelling with 3-D solid elements: (a) shaft element or superelement, and (b) basic continuum element.
The eight nodes define the mapping and therefore the boundary form of the quarter-annulus cross section. It is found that such an approximation is extremely accurate, and has a maximum error in the radial direction of about 1\% (Schwarz, 1988).

The straightforward formulation of displacement functions involves simple isoparametric elements. However, it is found that in order to achieve convergent results of bending responses, more elements must be employed though torsional and axial results have already been satisfactory. This behaviour indicates that bending characteristics are not well reflected by this isoparametric element formulation.

It is well known that the transverse displacements $u$ and $v$ contribute much more to bending modes than the axial displacement $w$. One might notice that the isoparametric element formulation can only satisfy $C^{0}$ continuity along the axial direction. This means that slopes or deflections may not be continuous. To possess $C^{1}$ continuity along the axial direction and high polynomials of $u$ and $v$, the displacement functions are expressed as

$$
\begin{aligned}
& u=\left\{g_{t}\right\}^{\mathrm{T}}\left[T_{t}\right]^{-1}\left\{\begin{array}{c}
u_{i} \\
\partial u_{i} / \partial z
\end{array}\right\}, \\
& v=\left\{g_{t}\right\}^{\mathrm{T}}\left[T_{t}\right]^{-1}\left\{\begin{array}{c}
v_{i} \\
\partial v_{i} / \partial z
\end{array}\right\}, \\
& w=\{g\}^{\mathrm{T}}[T]^{-1}\left\{w_{i}\right\},
\end{aligned}
$$

where

$$
\left\{g_{t}\right\}^{\mathrm{T}}=\left[\{h\}^{\mathrm{T}}, \zeta\{h\}^{\mathrm{T}}, \zeta^{2}\{h\}^{\mathrm{T}}, \zeta^{3}\{h\}^{\mathrm{T}}\right] .
$$

For transverse displacements, the transformation coefficient matrix $\left[T_{t}\right]$ will be dependent on the real geometry of the concerned element.

Kinetic and potential energies for each element can be expressed in terms of its nodal variables. Note that Hamilton's principle can be given by

$$
\int_{t_{1}}^{t_{2}}(\delta T-\delta V+\delta W) \mathrm{d} t=0
$$


where $\delta W$ is the virtual work done by internal and external generalized nodal forces.

Substitution of Eqs. (4)-(7) into Eq. (8) yields the equation of motion for the basic continuum element:

$$
\begin{aligned}
& {[M]\{\ddot{U}\}+[J(\Omega)]\{\dot{U}\}+\left[K_{r}(\Omega)\right]\{U\}} \\
& \quad=\{N(\Omega)\}+\{f\}+\{F\},
\end{aligned}
$$

where $[M],[J(\Omega)]$ and $\left[K_{r}(\Omega)\right]$ are the mass, gyroscopic and rotating stiffness matrices, respectively, $\{N(\Omega)\}$ involves centrifugal forces, $\{f\}$ stands for internal forces between adjacent elements, $\{F\}$ represents an external generalized force vector and $\{U\}$ denotes the nodal displacement and slope vector which is given by

$$
\{U\}=\left[\left\{u_{i}\right\}^{\mathrm{T}},\left\{\frac{\partial u_{i}}{\partial z}\right\}^{\mathrm{T}},\left\{v_{i}\right\}^{\mathrm{T}},\left\{\frac{\partial v_{i}}{\partial z}\right\}^{\mathrm{T}},\left\{w_{i}\right\}^{\mathrm{T}}\right]^{\mathrm{T}} .
$$

\subsection{Numerical Examples and Comparison with Beam Solutions and Measured Results}

For the above 3-D solid FE modelling, reduction procedures can be employed such as the continuous coordinate condensation (Craggs and Eckert, 1992) and the TMM based on FE models (Yu and Craggs, 1995b). The results for nonrotating tapered shafts $(\Omega=0)$ are shown first to yield insight into the accuracy and effectiveness of the proposed approach compared to others. Then the dynamic responses for a rotating uniform shaft are given and interesting behaviours are discussed.

Two special restricted cases, i.e., slender and stubby shafts are examined using the 3-D solid FE model. Here a slender shaft is characterized with $L=8 \mathrm{~m}$ in Fig. 3, and a stubby one with $L=0.8 \mathrm{~m}$. Compared with the Euler and Timoshenko beam solutions, the results for the two cases are given in Table I.

It can be seen that the 3-D solid FE model gives agreement with both the Euler and the Timoshenko beam theories for bending frequencies of a slender uniform shaft, and shows consistency with the Timoshenko beam solutions for a stubby shaft. However, when the shaft has a non-uniform cross section which can vary over its length, it is wrong to use the assumption in the simple beam theories that plane sections remain plane. In this case, an appropriate approach is to use a 3-D continuum model with the FE technique.

Vest and Darlow (1990) measured a free-free tapered shaft, as shown in Fig. 4. Since they found that tapered shafts cannot be correctly modelled using a standard beam theory, they tried to use some empirical formulas to correct the Young's modulus and therefore gave the corresponding results which are listed in Table II. This may not be a valid approach for different shafts whose geometry varies. Stephenson and Rouch (1993) used an axisymmetric element approach and obtained results as well.

Using the proposed 3-D subparametric element approach, the computed results are in the best

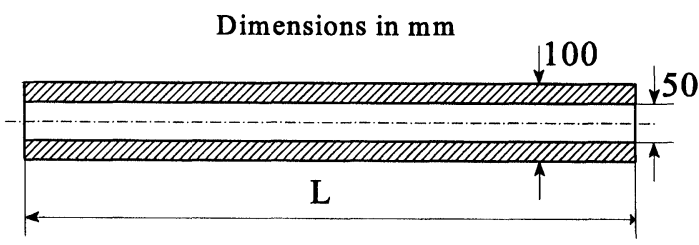

FIGURE 3 Uniform shaft.

TABLE I Bending frequencies $(\mathrm{Hz})$ of slender and stubby shafts using three different approaches

\begin{tabular}{cccccccc}
\hline Order & \multicolumn{3}{c}{ Slender shaft } & & \multicolumn{3}{c}{ Stubby shaft } \\
\cline { 2 - 3 } & Euler beam & Timoshenko beam & 3-D solid FE & & Euler beam & Timoshenko beam & 3-D solid FE \\
\hline 1 & 8 & 8 & 8 & & 787 & 744 & 744 \\
2 & 22 & 22 & 42 & & 4255 & 367 & 1875 \\
3 & 42 & 42 & 2281 & 3307 \\
\hline
\end{tabular}


TABLE II Free-free natural frequencies $(\mathrm{Hz})$ of tapered shaft with 8 and 12 superelements for a uniform and tapered section, respectively

\begin{tabular}{|c|c|c|c|c|c|c|c|}
\hline \multirow[t]{2}{*}{ Order } & \multicolumn{3}{|c|}{ Vest and Darlow (1990) } & \multirow{2}{*}{$\begin{array}{c}\text { Stephenson and Rouch (1993) } \\
\text { Axisymmetric FE } \\
\text { (bending) }\end{array}$} & \multicolumn{3}{|c|}{ Authors' 3-D solid FE } \\
\hline & $\begin{array}{l}\text { Measured } \\
\text { (bending) }\end{array}$ & $\begin{array}{l}\text { Beam elements } \\
\text { (bending) }\end{array}$ & $\begin{array}{l}\text { Modulus corrected } \\
\text { (bending) }\end{array}$ & & Bending & Torsional & Axial \\
\hline 1 & 1040 & $1072.1(3.1)$ & $1043.8(0.4)$ & $1035.9(-0.4)$ & $1050(0.96)$ & 5098 & 6216 \\
\hline 2 & 1643 & $1693.6(3.0)$ & $1658.7(0.9)$ & $1624.5(-1.1)$ & $1646(0.18)$ & 5454 & 8717 \\
\hline 3 & 4042 & $4161.3(2.9)$ & $4123.0(2.0)$ & $4068.6(0.7)$ & $4107(1.61)$ & 8087 & 13283 \\
\hline 4 & 5886 & $5970.0(1.4)$ & $5817.0(-1.2)$ & $5851.6(-0.6)$ & $5913(0.46)$ & & \\
\hline 5 & 7459 & $7943.0(6.5)$ & $7867.0(5.5)$ & $7533.0(1.0)$ & $7550(1.22)$ & & \\
\hline
\end{tabular}

Percent error from measured frequencies in parentheses.

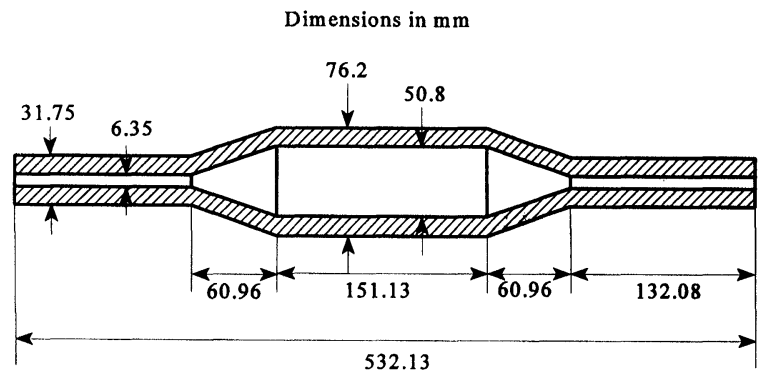

FIGURE 4 Tapered hollow shaft.

agreement with, and always above, the measured frequencies, as shown in Table II. For consistent FE modelling, computed natural frequencies should be always above the true values. The solutions obtained by the authors satisfied this criterion.

Natural frequencies of corresponding torsional and axial modes are also obtained using the same computer code, as shown in this table, though the corresponding measured results are not available. This is one of the merits of the 3-D solid FE model. In addition to bending motion, torsional, axial, and other possible modes are automatically included in this 3-D model, and do not need special addition as they would in beam models.

\section{SHAFT ORBITING}

Shaft orbiting is a very unique dynamic phenomenon which does not exist in other non-rotating vibrational systems. Natural frequencies, i.e., resonant orbiting frequencies, vary with shaft speed. There are two types of orbiting motion: forward and backward precessions. If any oscillation appears in the rotating frame (asynchronous orbiting), there will be dynamic stresses varying with time.

\subsection{Definition of Orbiting in the Rotating Frame}

Since the rotating frame is used to simulate the shaft motion, its rigid rotation will not be involved. The shaft can only have elastic displacements $u, v$, and $w$ in this frame. Due to Coriolis acceleration, there will be both $x$ and $y$ directional motions even though excitation or perturbation is only applied in either $x$ or $y$ direction. For a symmetric circular shaft as shown in Fig. 3, the orbiting motion can be described by Fig. 5 .

Figure 5 illustrates the orbiting motion of an arbitrary cross section. Line AB within this section will not have any rotational motion in the $x y$ plane. During one cycle of oscillation, this section has a translational motion along the $z$ axis although a small relative deformation within the section may be involved. It also undergoes axial vibrations as seen in the $y z$ and $x z$ planes.

It can be seen that in the rotating frame, the complicated shaft orbiting can be simply viewed as elastic translational motion if deflection is considered, as though the shaft were not rotating. Since the rotating frame is used throughout the analysis, the transverse displacements of the particles within 


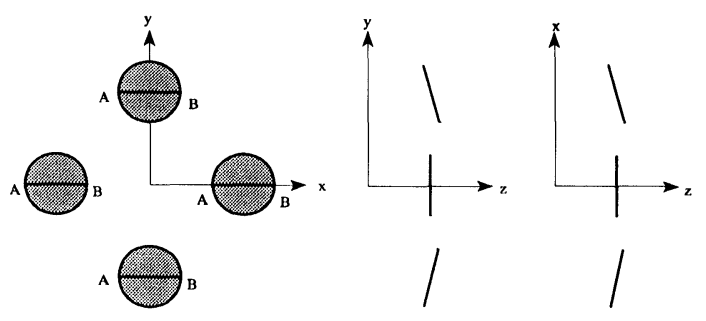

FIGURE 5 Shaft orbiting in the rotating frame.

the same cross section are almost the same when only bending behaviour is examined. If the fixed frame were employed, the displacements involving rigid and elastic motions would be different even within the same cross section. The axial oscillation of the shaft is also involved. Unlike a non-rotating shaft, it has a circular trajectory at resonance. The orbiting frequencies at resonance are just natural frequencies, which vary with rotational speed $\Omega$ when bending vibration is examined. This type of motion is called shaft orbiting, which includes nonresonant and resonant precessions.

Though trajectories at different nodal points within one cross section are not exactly the same using 3-D solid finite elements, it has been found that transverse displacements within the same cross section are very close in this case regardless of very different axial displacements. Therefore, the average value of the transverse displacements at different nodal points is adopted to represent the trajectory of this cross section.

The orbiting direction could be the same as or opposite to that of the rotational speed. If the dynamic responses in $x$ and $y$ directions of the rotating frame are expressed in the complex form

$$
u=A \mathrm{e}^{\mathrm{i}\left(\omega t+\varphi_{u}\right)}, \quad v=B \mathrm{e}^{\mathrm{i}\left(\omega t+\varphi_{v}\right)},
$$

where $\omega$ is the forcing frequency in the rotating frame, then the precessional angle relative to the $x$-axis is given by

$$
\theta=\tan ^{-1}\left(\frac{\operatorname{Re}(v)}{\operatorname{Re}(u)}\right)
$$

It follows that

$\omega_{\text {rot }}=\frac{\mathrm{d} \theta}{\mathrm{d} t}=\omega \frac{A B \sin \left(\varphi_{u}-\varphi_{v}\right)}{A^{2} \cos ^{2}\left(\omega t+\varphi_{u}\right)+B^{2} \cos ^{2}\left(\omega t+\varphi_{v}\right)}$.

The orbiting will be counterclockwise if $\varphi_{u}-\varphi_{v}$ is between 0 and $\pi$, and otherwise will be clockwise. There is a special case where $A=B$ and $\left|\varphi_{u}-\varphi_{v}\right|=$ $\pi / 2$. In this case, the orbiting frequency $\omega_{\text {rot }}$ will be constant all the time.

It is quite common for rotordynamic analysts to use orbiting frequencies in the fixed reference frame. A simple formula can be given by

$$
\omega_{\mathrm{fix}}=\omega_{\mathrm{rot}}+\Omega
$$

where $\Omega$ is assumed to be positive.

If $\omega_{\text {rot }}$ has the same rotational direction as $\Omega$, then it is said to have forward orbiting in the rotating frame; if it has the opposite direction, then it is said to have backward orbiting in the rotating frame. The direction and frequency magnitude of the corresponding orbiting in the fixed frame are then determined by Eq. (14). It can be found that backward orbiting in the rotating frame can become forward orbiting in the fixed frame.

\subsection{Forced Response: Forward and Backward Orbiting}

A simple uniform shaft, as shown in Fig. 4, is used here as an example to examine dynamic responses under an assumed excitation with a forcing frequency in the rotating frame.

Figure 6 shows a case where an exciting force is applied to the left end of the shaft in the $x$-direction of the rotating frame. Figure 6(a) gives $x$-directional and $y$-directional responses, which in terms of Eq. (11) generally yield elliptic trajectories as shown in Fig. 6(b).

It can be seen that unlike non-rotating bodies, an excitation on the shaft in the $x$-direction causes transverse displacements in not only the $x$-direction but also the $y$-direction. This is due to the rotational 


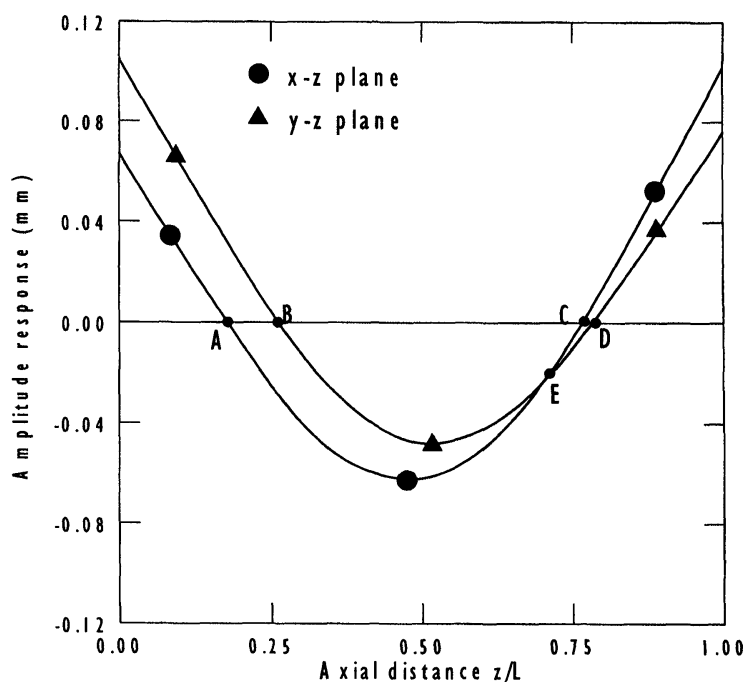

(a)
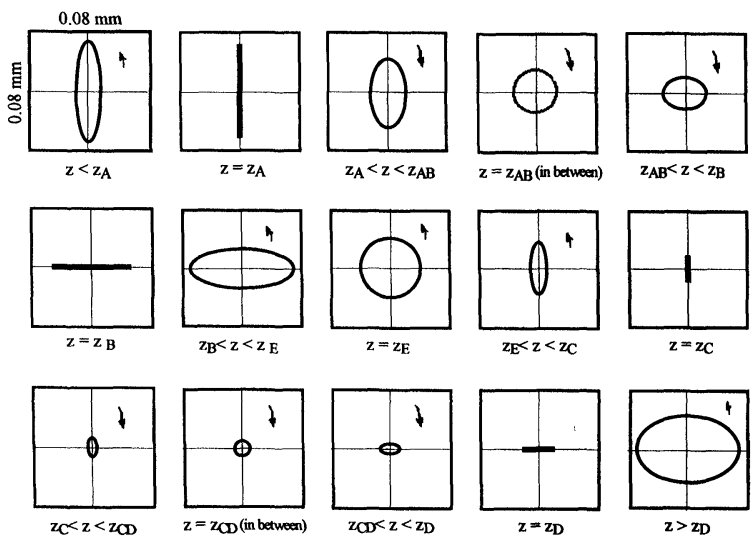

(b)
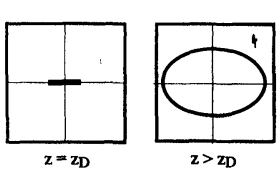

FIGURE 6 Forced response when an exciting force with an amplitude of $1 \mathrm{kN}$ and a frequency of $495 \mathrm{~Hz}$ was applied to the left end in $x$-direction of the rotating frame with speed of $1570.8 \mathrm{rad} / \mathrm{s}$ : (a) transverse displacement vs. axial distance, and (b) orbits from the left to the right end of the shaft in the rotating frame. speed which leads to the Coriolis acceleration. In Fig. 6(a) the positive amplitude of the $x$-directional response corresponds to phase angle $\varphi_{u}=0$ and that of the $y$-directional response corresponds to $\varphi_{v}=-\pi / 2$. The $x$-directional responses have a phase angle of either 0 or $-\pi$, and $y$-directional responses have a phase angle of either $-\pi / 2$ or $\pi / 2$.

Near the left end, orbits can be seen to be elliptic after the superposition of two orthogonal responses, with forward orbiting as defined in Eq. (13). At the location $\mathrm{A}$, the transverse displacement in the $x z$ plane becomes zero, thus the orbit is a straight line along the $y$-direction. Between locations $\mathrm{A}$ and $\mathrm{B}$ of the shaft, there exists backward orbiting in terms of Eq. (13). There is also a location where amplitudes of $x$ and $y$ directional responses are the same, thus making the backward trajectory circular. At the location B, the orbit becomes a straight line in the $x$-direction. Then after location $\mathrm{B}$, orbits become forward again until the location $\mathrm{E}$ at which the two amplitude curves intersect. Obviously the orbit at the location $\mathrm{E}$ is circular with forward orbiting. Orbits are backward between locations $\mathrm{C}$ and $\mathrm{D}$. Near the right end of the shaft after the location $\mathrm{D}$, orbits become forward again. It can be seen that relative orbits are still forward as shown in Fig. 7.

When the forcing frequency is increased to $505 \mathrm{~Hz}$, the characteristics of the response are totally different, as shown in Fig. 8. Though only one exciting force is applied to the left end of the shaft, the response is symmetric to the midspan for that frequency. The orbiting is purely forward with circular orbits all along the shaft. The amplitude of the response is extremely big, compared with other
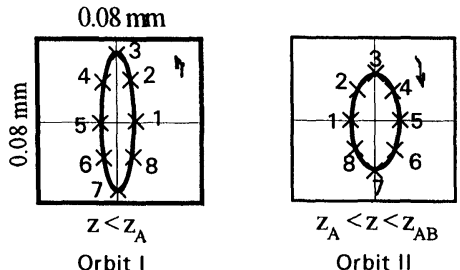
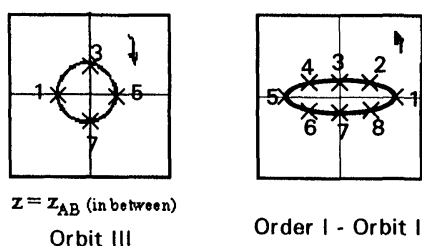

Order I - Orbit II

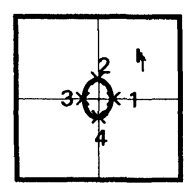

Orbit II - Orbit III

FIGURE 7 Relative orbit. 


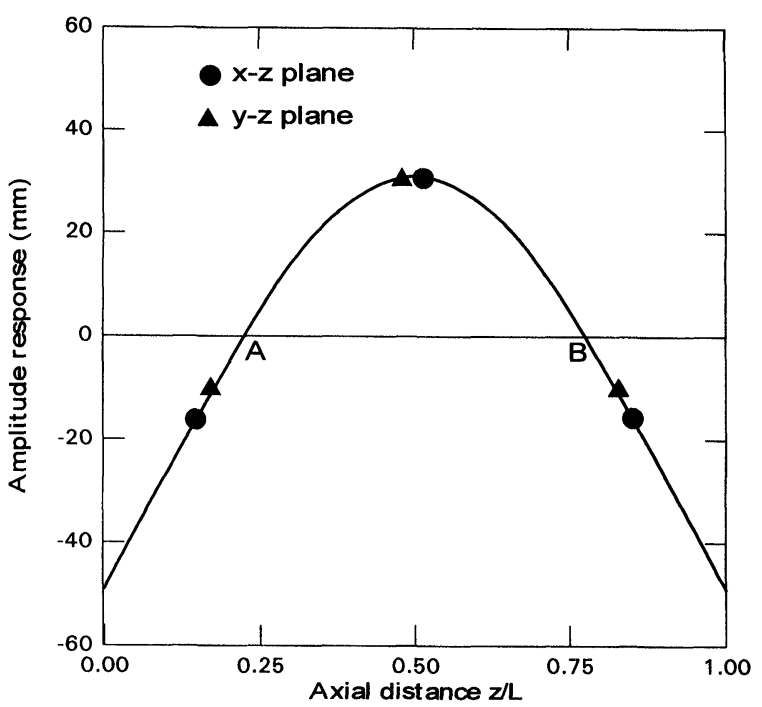

(a)
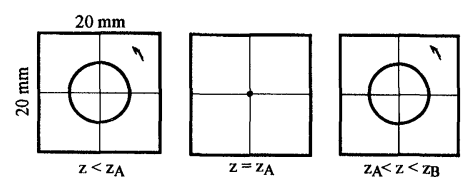

(b)
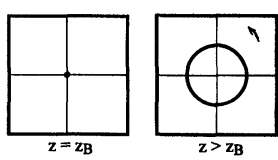

FIGURE 8 Resonant response when an exciting force with an amplitude of $1 \mathrm{kN}$ and a frequency of $505 \mathrm{~Hz}$ was applied to the left end in $x$-direction of the rotating frame with speed of $1570.8 \mathrm{rad} / \mathrm{s}$ : (a) transverse displacement vs. axial distance, and (b) orbits from the left to the right end of the shaft in the rotating frame.

forcing frequencies. This is the case in which a forcing frequency reaches the resonant forward orbiting frequency.

With the forcing frequency being increased, response orbits become elliptic again for most sections. Forward orbiting coexists with backward orbiting. When the frequency is far away from the previous forward orbiting frequency, backward orbiting becomes dominant. At the frequency of $930 \mathrm{~Hz}$, the forced response and its trajectories are shown in Fig. 9. When the forcing frequency increases to $980 \mathrm{~Hz}$, i.e., a resonant backward orbiting frequency, the shaft has purely backward orbiting responses along the whole shaft, as shown in Fig. 10.

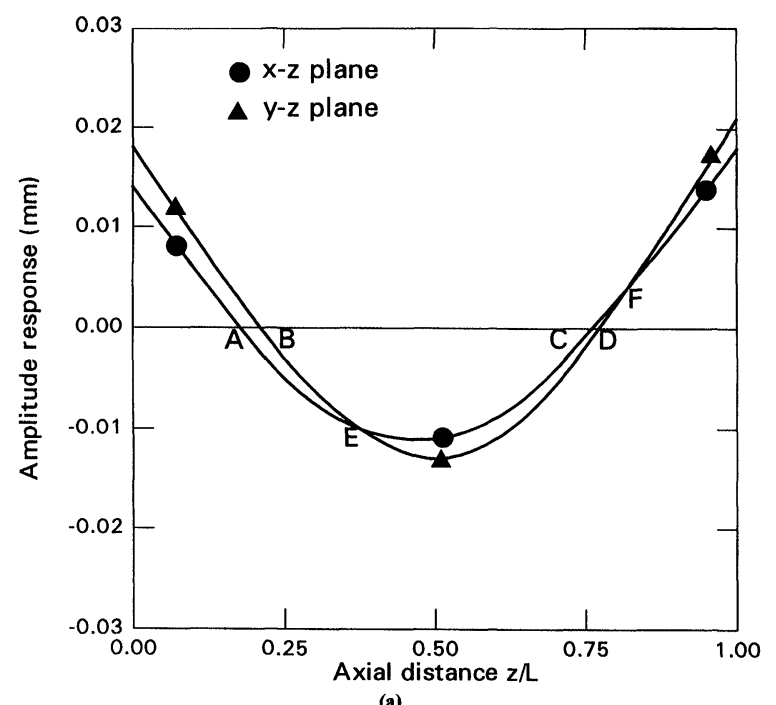

(a)
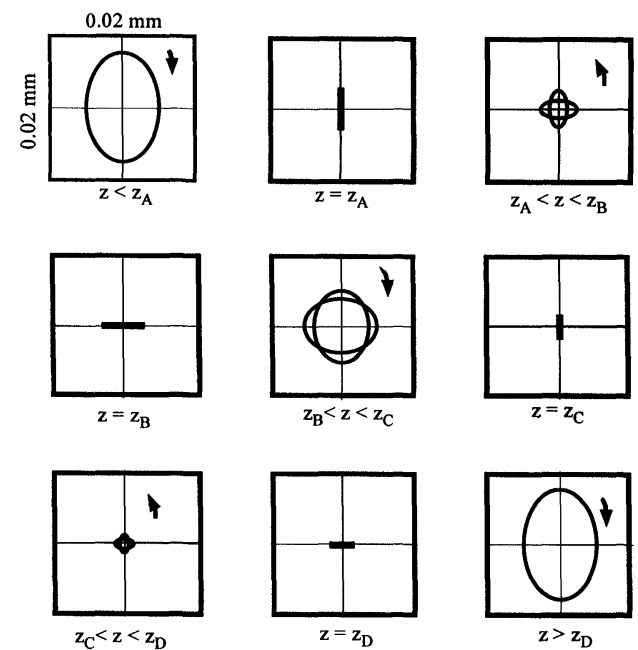

(b)

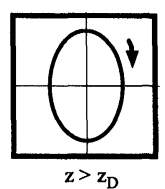

FIGURE 9 Forced response when an exciting force with an amplitude of $1 \mathrm{kN}$ and a frequency of $930 \mathrm{~Hz}$ was applied to the left end in $x$-direction of the rotating frame with speed of $1570.8 \mathrm{rad} / \mathrm{s}$ : (a) transverse displacement vs. axial distance, and (b) orbits from the left to the right end of the shaft in the rotating frame.

One can see that the characteristics of the shaft orbiting motion depend on the forcing frequency in the rotating frame. At non-resonant frequencies, forward and backward orbiting will coexist along the shaft. When the response is close to forward or backward orbiting, the relative orbit (Muszynska, 


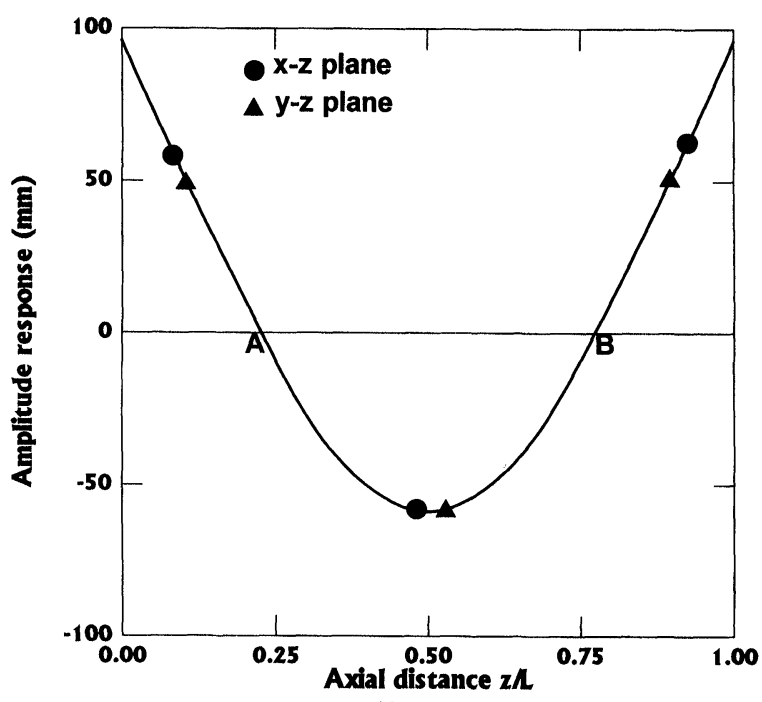

(a)
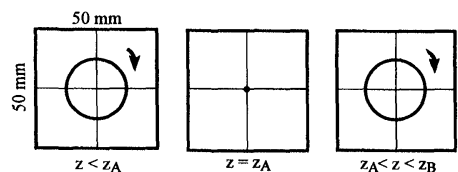

(b)
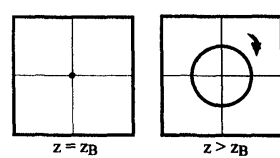

FIGURE 10 Resonant response when an exciting force with an amplitude of $1 \mathrm{kN}$ and a frequency of $980 \mathrm{~Hz}$ was applied to the left end in $x$-direction of the rotating frame with speed of $1570.8 \mathrm{rad} / \mathrm{s}$ : (a) transverse displacement vs. axial distance, and (b) orbits from the left to the right end of the shaft in the rotating frame.

1996; Muszynska et al., 1996) can identify it. Using Eq. (14) in which relative $u$ and $v$ are used, relative orbits within either the left or the right half side of the shaft are forward at the frequency of $495 \mathrm{~Hz}$, and backward at the frequency of $930 \mathrm{~Hz}$, which can be concluded from Figs. 7 and 9. If two orthogonal bending amplitude curves are shown in Fig. 11, any relative orbit in the shaft will be forward at that frequency.

Authors believe that the orbiting nature can be determined by the amplitude and phase curves in the two orthogonal bending planes. Figures 7, 9 and 11 have regions of $\mathrm{AB}$ and $\mathrm{CD}$, thus making forward and backward precessions mixed up along the shaft. Points A, B, C, and D are the critical points where the transition between forward and

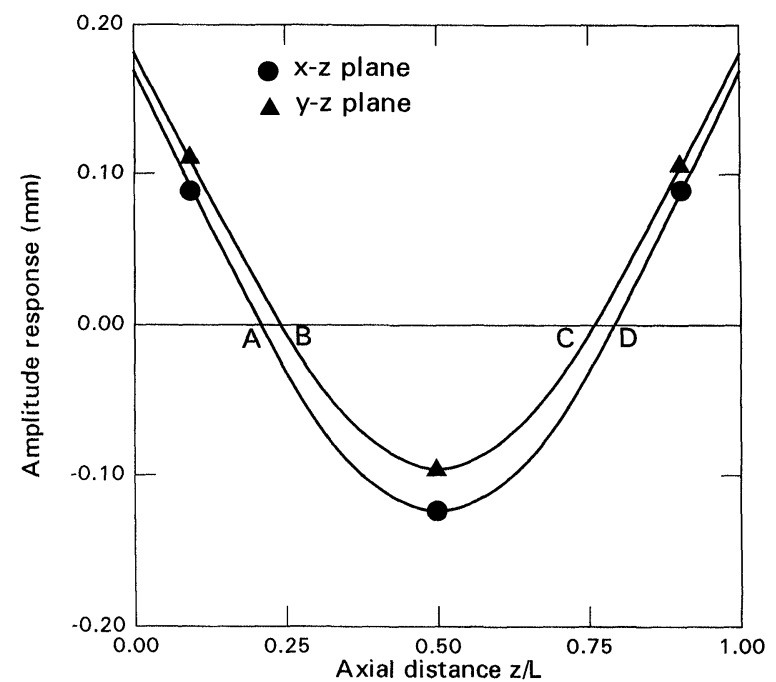

FIGURE 11 Forced response due to two exciting forces applied to the two ends. Other conditions were the same as those in Fig. 6.

backward precessions is located. At these points, the orbiting becomes a straight line. The orbiting is the superposition of the responses in the two orthogonal bending planes.

\subsection{Resonant Orbiting Frequencies}

Resonant orbiting frequencies are defined as the natural frequencies of the rotating shaft. As indicated above, they correspond to infinitely large responses for undamped rotating shafts. In reality, a peak response frequency is considered to be the resonant frequency when a sweeping technique is used with a certain frequency step. At resonance, there is either forward or backward orbiting all along the shaft, with the circular trajectories for a uniform shaft, as shown in Figs. 8 and 10.

It is believed that for a uniform shaft, a 3-D solid continuum model will yield results close to the solutions with the Timoshenko beam model, as shown in Table III. The shaft size is the same as that in Fig. 4.

It can be seen that for an $i$ th mode, there exist both forward and backward orbiting frequencies for the given rotational speed instead of only one 
TABLE III Forward and backward orbiting natural frequencies $(\mathrm{Hz})$ for $\Omega=1570.8 \mathrm{rad} / \mathrm{s}$

\begin{tabular}{lccc}
\hline $\begin{array}{l}\text { Mode type } \\
\mathrm{F}_{i}^{\dagger} \mathrm{B}_{i}^{\dagger}\end{array}$ & $\begin{array}{c}\text { 3-D solid FE results } \\
\text { (in rotating frame) }\end{array}$ & $\begin{array}{c}\text { 3-D solid FE results } \\
\text { (in fixed frame) }\end{array}$ & $\begin{array}{c}\text { Timoshenko beam solutions* } \\
\text { (Zhu and Han, 1992) (in fixed frame) }\end{array}$ \\
\hline $\mathrm{F}_{1}$ & 505 & 755 & 757 \\
$\mathrm{~B}_{1}$ & 980 & 730 & 731 \\
$\mathrm{~F}_{2}$ & 1645 & 1895 & 1889 \\
$\mathrm{~B}_{2}$ & 2101 & 1851 & 1845 \\
$\mathrm{~F}_{3}$ & 3083 & 3333 & 3308 \\
$\mathrm{~B}_{3}$ & 3529 & 3279 & 3254 \\
\hline
\end{tabular}

‘ $\mathrm{F}$ " and " $\mathrm{B}$ " are referred to forward and backward precessions, respectively. Subscript " $i$ " stands for a mode order, $i=1,2,3$. *A shear coefficient of 0.6202 is adopted.

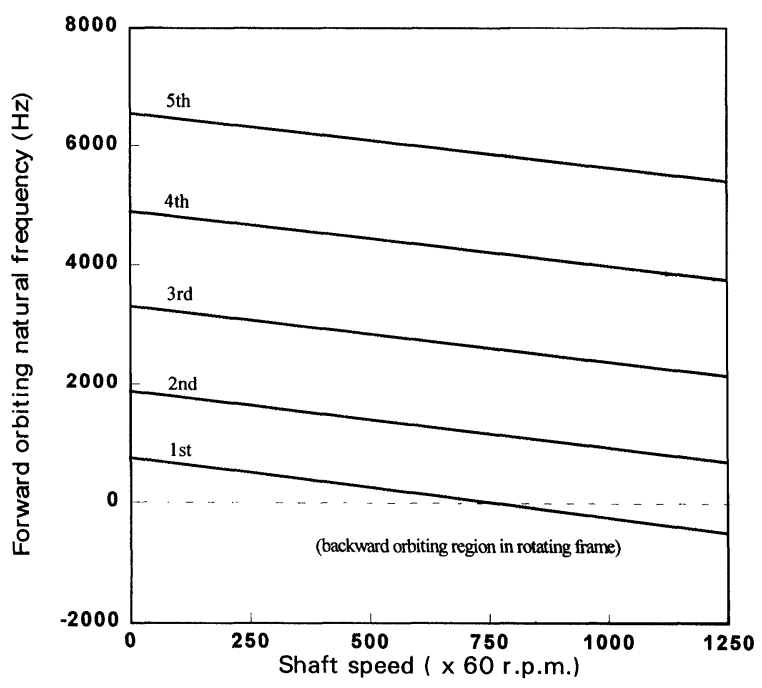

FIGURE 12 Forward orbiting natural frequency in the rotating frame vs. shaft speed.

frequency for a non-rotating case. This confirms that gyroscopic effects have been consistently included using the proposed 3-D solid FE modelling. All these values can be determined by computer simulation through the developed code which uses the reduction procedures. Orbiting directions and frequencies in the fixed frame can also be determined using Eqs. (11)-(14).

The effect of rotational speeds on resonant orbiting frequencies has been examined for the same shaft. The frequencies obtained from the analysis can be directly expressed in the rotating frame. Figure 12 gives the forward orbiting frequencies in the rotating frame. In this way,

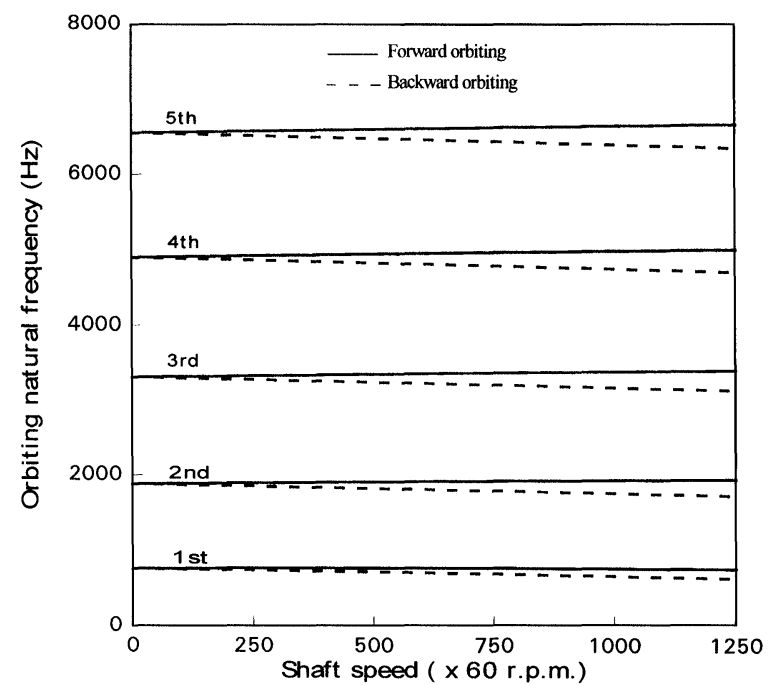

FIGURE 13 Forward and backward orbiting natural frequency in the fixed frame vs. shaft speed.

critical speeds can be determined when these frequency curves reach zero, as shown in Fig. 12 where the first critical speed is $764 \mathrm{cps}$. Critical speeds can also be obtained by $\left[K_{r}(\Omega)\right]$ term in Eq. (9).

Synchronous orbiting corresponds to zero orbiting frequency in the rotating frame, which means no relative oscillation within the shaft. Therefore, no time-varying dynamic stresses appear at a given speed. However, the shaft can be bowed severely at critical speeds. If the orbiting speed changes within a rotation, this motion is only an approximated synchronous orbiting which still causes dynamic stresses. 
If resonant orbiting frequencies are expressed in the fixed frame, the corresponding magnitudes can be determined by Eq. (14). Conventionally, backward orbiting is specified without adding a "-" sign. Figure 13 shows the first five order orbiting frequencies vs. the shaft speed. As the shaft speed increases, forward orbiting frequencies increase and backward orbiting frequencies decrease in the fixed frame. In this figure, the solid line denotes the forward orbiting, and the broken line represents the backward orbiting.

\section{CONCLUSIONS}

A 3-D solid FE model is proposed in which not only bending but also torsion, axial and other possible modes are included automatically. In the special restricted cases of a slender or stubby uniform shaft, this model shows consistency with the Euler and/or Timoshenko beam theories when bending frequencies are examined. However, when the shaft has a non-uniform cross section varying with its length such as tapered shafts, a 3-D solid FE model should be used to accurately depict the deformation. It is shown that the computed results for a tapered shaft are in very good agreement with the measured data. Reduction procedures can be implemented to allow a small matrix operation for this viable model with a beam-like structure.

The rotating frame is used which facilitates the FE modelling and gives a clear view of the shaft orbiting of bending vibration. The orbiting behaviour has been examined under harmonic excitations in the rotating frame. Forward and backward precessions could coexist along the shaft if observed in the rotating frame. When forcing frequencies reach resonant orbiting frequencies, the orbiting become either purely forward or purely backward. This orbiting nature in the rotating frame is believed to be reported for the first time, which may aid rotordynamic analysis. The authors view this peculiar phenomenon as the consequence of the combination of two different deflection curves in the two orthogonal bending planes.

\section{Acknowledgments}

Operating grants from the Natural Science and Engineering Research Council of Canada held by A. Craggs and A. Mioduchowski are greatly appreciated.

\section{References}

Akella, S. and Craggs, A. (1986) Modifications to a Timoshenko beam-shaft finite element to include internal disks and changes in cross-section, Journal of Sound and Vibration, 106, 227-239.

Choi, S.H., Pierre, C. and Ulsov, A.G. (1992) Consistent modelling of rotating Timoshenko shafts subject to axial loads, ASME, Journal of Vibration and Acoustics, 114, 249-259.

Craggs, A. and Eckert, W.F. (1992) A reduction procedure for finite element models of beams and shafts, Journal of Sound and Vibrations, 156, 559-570.

Genta, G. and Tonoli, A. (1996) A harmonic finite element for the analysis of flexural, torsional and axial rotordynamic behaviour of discs, Journal of Sound and Vibration, 196, 19-43.

Geradin, M. and Kill, N. (1984) A new approach to finite element modelling of flexible rotors, Engineering Computations, 1, 52-64.

Muszynska, A. (1996) Forward and backward procession of a vertical anisotropically supported rotor, Journal of Sound and Vibration, 192, 207-222.

Muszynska, A., Hatch, C.T. and Bently, D.E. (1996) Dynamics of anisotropically supported rotors, Proceedings of ISROMAC-6, pp. $1-10$.

Nelson, H.D. (1980) A finite rotating shaft element using Timoshenko beam theory, ASME Journal of Mechanical Design, 102, 793-803

Rouch, K. and Kao, J.S. (1979) A tapered beam finite element for rotordynamics analysis, Journal of Sound and Vibration, 66, 119-140.

Senthilnathan, N.R. and Lee, K.H. (1992) Some remarks on Timoshenko beam theory, ASME Journal of Vibration and Acoustics, 114, 495-497.

Schwarz, H.R. (1988) Finite Element Methods, Academic Press.

Stephenson, R.W. and Rouch, K.E. (1993) Modelling rotating shafts using axisymmetric solid finite elements with matrix reduction, ASME Journal of Vibration and Acoustics, 115, 484-489.

Stephenson, R.W., Rouch, K.E. and Arora, R. (1989) Modelling of rotors with axisymmetric solid harmonic elements, Journal of Sound and Vibration, 131, 431-443.

Timoshenko, S.P. (1922) On the transverse vibrations of bars of uniform cross-section, Philosophical Magazine, 43, 125-131.

Vance, J.M. (1988) Rotor Dynamics of Turbomachinery, John Wiley \& Sons, New York.

Vest, T.A. and Darlow, M.S. (1990) A modified conical beam element based on finite element analysis: experimental correlations, ASME, Journal of Vibration and Acoustics, 112, $350-354$.

Yu, J. and Craggs, A. (1995a) Dynamic analysis of hollow shafts with 3-D solid finite elements, Proceedings of the 15th Canadian Congress of Applied Mechanics, Victoria, British Columbia, pp. 358-359. 
Yu, J. and Craggs, A. (1995b) Transfer matrix method for finite element models of a chain-like structure under harmonic excitations, Journal of Sound and Vibration, 187, $169-175$.

Yu, J. and Craggs, A. (1997) 3-D solid finite element modelling of rotating shafts, Proceedings of the 15th
International Modal Analysis Conference, Orlando, Florida, pp. $1488-1494$.

$\mathrm{Zu}$, Jean W.Z. and Han, Ray P.S. (1992) Natural frequencies and normal modes of a spinning Timoshenko beam with general boundary conditions, ASME Journal of Applied Mechanics, 59, S197-S204. 


\section{ait \\ ENERGY MATERIALS}

M A N E Y publishing

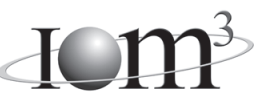

\section{Materials Science \& Engineering for Energy Systems}

Maney Publishing on behalf of the Institute of Materials, Minerals and Mining

The Institute of Materials, Minerals \& Mining

Economic and environmental factors are creating ever greater pressures for the efficient generation, transmission and use of energy. Materials developments are crucial to progress in all these areas: to innovation in design; to extending lifetime and maintenance intervals; and to successful operation in more demanding environments. Drawing together the broad community with interests in these areas, Energy Materials addresses materials needs in future energy generation, transmission, utilisation, conservation and storage. The journal covers thermal generation and gas turbines; renewable power (wind, wave, tidal, hydro, solar and geothermal); fuel cells (low and high temperature); materials issues relevant to biomass and biotechnology; nuclear power generation (fission and fusion); hydrogen generation and storage in the context of the 'hydrogen economy'; and the transmission and storage of the energy produced.

As well as publishing high-quality peer-reviewed research, Energy Materials promotes discussion of issues common to all sectors, through commissioned reviews and commentaries. The journal includes coverage of energy economics and policy, and broader social issues, since the political and legislative context influence research and investment decisions.

\section{CALL FOR PAPERS}

Contributions to the journal should be submitted online at http://ema.edmgr.com

To view the Notes for Contributors please visit: www.maney.co.uk/journals/notes/ema

Upon publication in 2006, this journal will be available via the Ingenta Connect journals service. To view free sample content online visit: www.ingentaconnect.com/content/maney

For further information please contact:

Maney Publishing UK

Tel: +44 (0)113 2497481 Fax: +44 (0)1132486983 Email: subscriptions@maney.co.uk

or

Maney Publishing North America

Tel (toll free): 8662975154 Fax: 6173546875 Email: maney@maneyusa.com

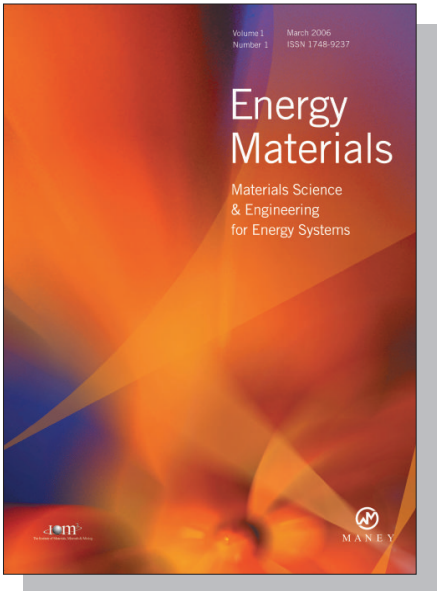

EDITORS

Dr Fujio Abe

NIMS, Japan

Dr John Hald, IPL-MPT, Technical University of Denmark, Denmark

Dr R Viswanathan, EPRI, USA

\section{SUBSCRIPTION INFORMATION}

Volume 1 (2006), 4 issues per year

Print ISSN: 1748-9237 Online ISSN: 1748-9245

Individual rate: $£ 76.00 / U S \$ 141.00$

Institutional rate: $£ 235.00 /$ US $\$ 435.00$

Online-only institutional rate: $£ 199.00 / U S \$ 367.00$

For special $\mathrm{IOM}^{3}$ member rates please email

subscriptions@maney.co.uk

\section{For further information or to subscribe online please visit www.maney.co.uk}



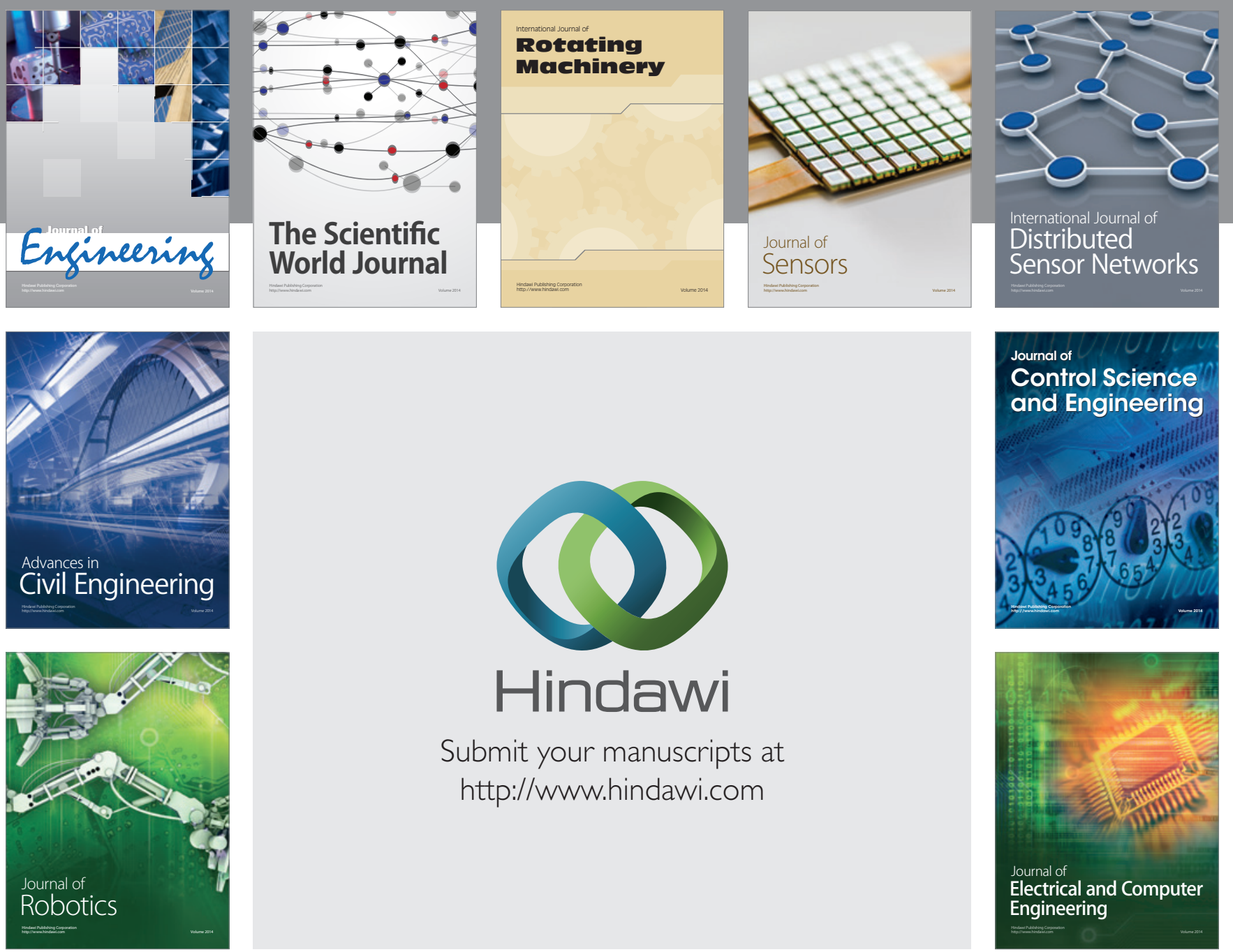

Submit your manuscripts at

http://www.hindawi.com
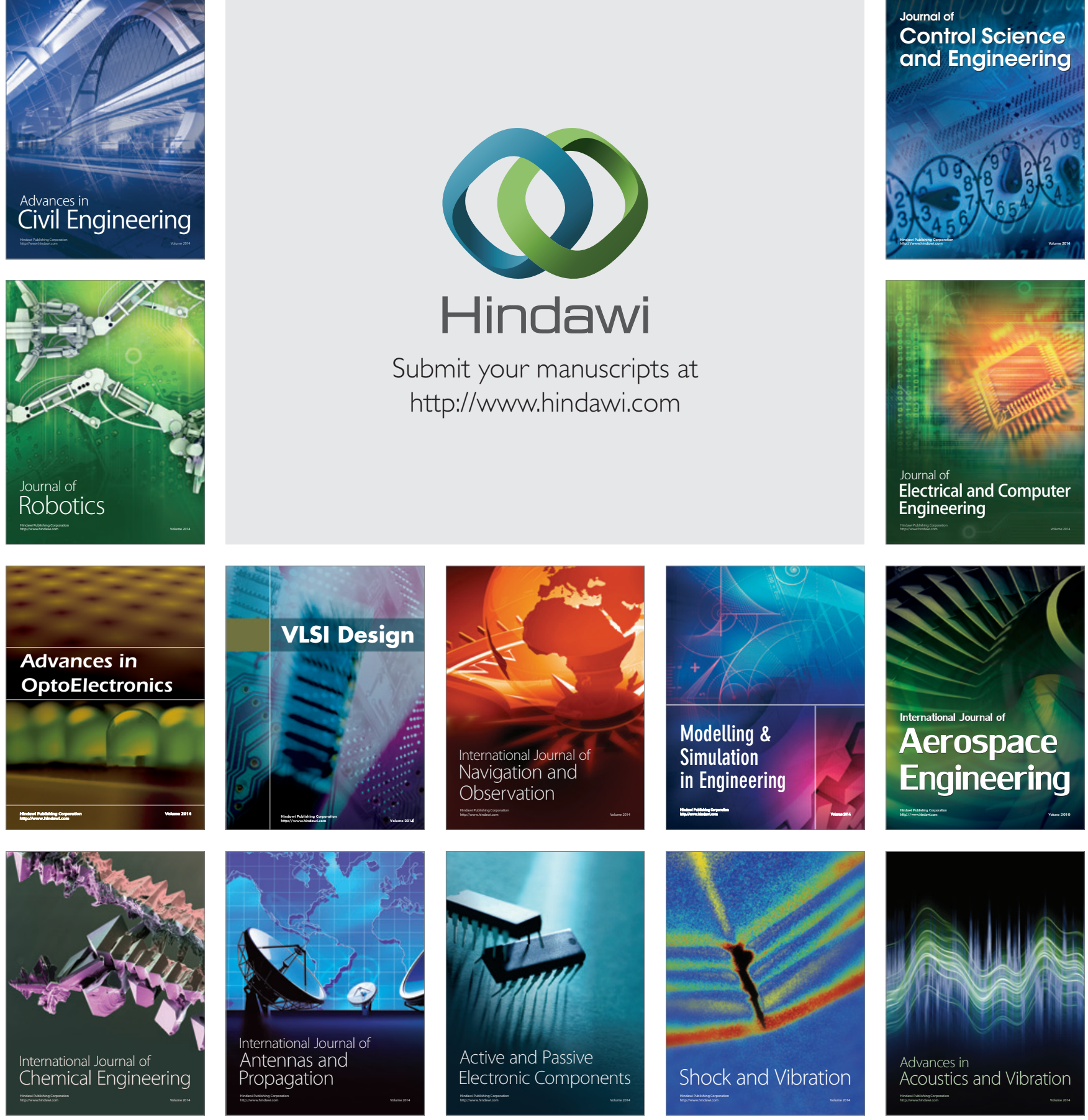